\title{
Syndecan-1 and Outcome of Ambulatory Patients with Stable Heart Failure
}

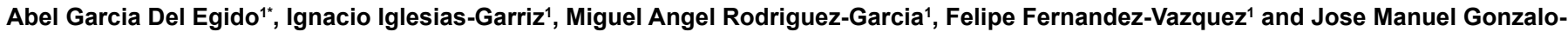 \\ Orden $^{2}$ \\ ${ }^{1}$ Cardiology Department, University Hospital of Leon, Leon, Spain \\ ${ }^{2}$ Institute of Biomedicine-IBIOMED, University of Leon, Leon, Spain
}

\begin{abstract}
Background: Syndecan-1 is a major component of the glycocalyx, a carbohydrate-rich layer lining the vascular endothelium. Endothelial glycocalyx contributes to the permeability of the vascular endothelium and could be a biomarker of clinical utility in patients with heart failure. We sought to investigate the utility of blood levels of syndecan-1 in the prognostic assessment of stable heart failure patients.

Methods: Ninety-five patients with stable heart failure (8 with preserved ejection fraction) and 24 matched controls were included. Blood concentrations of syndecan-1 were measured by ELISA. The end-point of the study was the combined of cardiovascular death and rehospitalization for heart failure. Syndecan-1 values were $\log _{2}$ transformed to obtain a normal distribution.

Results: The patient group had higher levels of the biomarker: 3.74 (DE 1.53) ng/mL vs. 2.84 (DE 1.7) ng/mL, $p=0.01 . \log _{2}$ (syndecan-1) did not change in patients according to their functional class $(p=0.543) . \log _{2}(\operatorname{syndecan}-1)$ was linearly related to some inflammatory cytokines: IL-1 $(r=0.47, p=0.009)$ and IL-6 $(r=0.44, p<0.001)$. After a follow-up of 544 days (interquartile range 285-688 days), we did not find differences in the end-point $(p=0.857$ ) by segregating patients into two groups (those with $\log _{2}$ (syndecan-1) above and below the median value). However, in a multivariate Cox proportional risk model, blood concentrations of $\log _{2}$ (syndecan-1) had a lower risk of mortality and rehospitalization: $\mathrm{HR}=0.64(0.45-0.92), \mathrm{p}=0.015$.
\end{abstract}

Conclusion: Higher blood levels of syndecan-1 are independently associated with a lower probability of cardiovascular death and hospitalization for heart failure in the mid-term follow-up.

Keywords: Diagnosis; Glycocalyx; Heart failure; Prognosis; Syndecan-1

Abbreviations: Hs-CRP: High-sensitivity C-Reactive Protein; HsTn: High-sensitivity Troponin; HR: Hazard Ratio; IL- $1 \beta$ : Interleukin $1 \beta$; IL6: Interleukin 6; NT-proBNP: N-terminal prohormone Brain Natriuretic Peptide

\section{Introduction}

Heart failure is a prevalent disease that portends a high mortality and morbidity rate. The correct evaluation of these patients is of paramount importance in order to reduce mortality, hospitalizations, and costs associated with the disease. However, the available information shows that clinical assessment of heart failure is inaccurate, particularly in some subsets of patients, such as women, obese patients, and the elderly $[1,2]$ and, as such, new tools are needed to enhance the appraisal of these patients.

Circulating cardiac biomarkers in patients with heart failure can be a valuable diagnostic and prognostic tool for the clinician. These can be divided into three groups: neurohormonal mediators, markers of myocyte injury-remodeling (including extracellular matrix components, mainly proteoglycans), and indicators of systemic inflammation [3,4]. Glycocalyx is a carbohydrate-rich layer lining the vascular endothelium. Endothelial glycocalyx is an important determinant of vascular permeability and could be responsible of the translation of biomechanical forces into biochemical signals to the endothelial cells and, as such, could be a consistent biomarker in heart failure. For example, its role in the regulation of interstitial edema, a hallmark feature of heart failure, has been described [5]. The thickness and chemical composition of the glycocalyx is in equilibrium with the flowing blood, and enzymatic or shear forces can shed it from the endothelium. Proteoglycans (syndecan-1 to 4) are the most important components of the endothelial glycocalyx, and the ecto-domain of syndecan-1, the major syndecan component of epithelial cells, has been shown to shed into the vascular system and is measurable in plasma [6]. The information provided by this molecule in patients with heart failure is uncertain. One study reported that syndecan-1 is associated with fibrotic and remodeling biomarkers and with outcomes in the subset of patients with heart failure and preserved ejection fraction, but not with inflammatory markers [7]. Interestingly, a possible protective effect of syndecan-1 shedding in stable patients has been suggested [7].

For these reasons, we hypothesized that syndecan- 1 could be a useful biomarker to evaluate the outcomes in patients with stable heart failure.

\section{Methodology}

\section{Patients and study design}

The trial was conducted as a prospective cohort study. Patients of at

*Corresponding author: Abel García Del Egido, Servicio De Cardiologia, Cardiology Department, University Hospital of Leon, C/Altos de Nava s/n, 24071, Leon, Spain, Tel: +346465380 59; E-mail: abelgarciadelegido@hotmail.com

Received September 03, 2018; Accepted September 29, 2018; Published October 04, 2018

Citation: Egido AGD, Iglesias-Garriz I, Rodriguez-Garcia MA, Fernandez-Vazquez F, Gonzalo-Orden JM (2018) Syndecan-1 and Outcome of Ambulatory Patients with Stable Heart Failure. J Mol Biomark Diagn 9: 401. doi: 10.4172/2155-9929.1000401

Copyright: (c) 2018 Egido AGD, et al. This is an open-access article distributed under the terms of the Creative Commons Attribution License, which permits unrestricted use, distribution, and reproduction in any medium, provided the original author and source are credited. 
least 18 years of age were diagnosed with heart failure, irrespective of their left ventricular ejection fraction, if they met the following criteria:

1) Dyspnea on effort (at least class II of the New York Heart Association),

2) Previous hospitalization for heart failure with high levels of NTproBNP ( $>400 \mathrm{pg} / \mathrm{mL}$ ), or echocardiographic left ventricular ejection fraction $<40 \%$ or echocardiographic left ventricular ejection fraction $\geq 40 \%$ with at least two of the following abnormalities: Left ventricular hypertrophy, left atrium enlargement $\left(>34 \mathrm{~mL} / \mathrm{m}^{2}\right)$, Doppler assessment with E/e' ratio >13 (e': mean tissue Doppler diastolic velocity of the lateral and medial mitral annulus), atrial fibrillation, velocity of the tricuspid regurgitation $>3 \mathrm{~m} / \mathrm{s}$, and

3) at least 1 determination of NT-proBNP $>125 \mathrm{pg} / \mathrm{mL}$ with the patient in a stable clinical condition.

Exclusion criteria were:

1) Moderate or severe aortic valvulopathy or mitral stenosis.

2) Moderate or severe lung disease.

3) Anemia with hemoglobin concentration below $10 \mathrm{~g} / \mathrm{dL}$.

4) Chronic renal disease, stages 4 or 5 .

5) Life expectancy of less than 1 year related to associated comorbidities.

6) Refusal to sign the informed consent.

We deemed a patient to be in a stable clinical condition if he/she met all the following criteria:

1) No admission for heart failure for at least 4 months before inclusion in the study.

2) No change in diuretic medications at least 1 month before the inclusion.

3) No progression of symptoms reported from the patient.

Ninety-five consecutive patients were selected for the analysis and twenty-four healthy controls were included to obtain the normal range blood levels of syndecan-1.

The study complies with the local ethics committee, and all the included patients provided written informed consent.

\section{Procedures}

On the day of the inclusion, patients received a thorough clinical evaluation, including an electrocardiogram and transthoracic echocardiogram to evaluate ejection fraction (Simpson's rule) and left ventricular diastolic function. The severity of mitral regurgitation was evaluated by an echocardiographist with a high level of expertise in the evaluation of valvular abnormalities and categorized into three groups: mild or absent, moderate, and severe. Left atrium volume was measured from the four-chamber apical view by means of the area-length method.

A blood sample was drawn on the first day of inclusion to measure the blood levels of these biomarkers: high-sensitivity troponin T (hsTn), NT-proBNP, ST2, interleukin-1 $\beta$, interleukin-6, high-sensitivity C-reactive protein (hsCRP), and syndecan-1. With the exception of hsTn and NT-proBNP, the obtained plasma was frozen at $-80^{\circ} \mathrm{C}$ and stored until analysis was performed by enzyme-linked immunosorbent assay (ELISA) (kits provided by Diaclone BioNova Scientific, Madrid, Spain and Duoset R\&D systems, Minneapolis, USA), according to the manufacturer's recommendations.

\section{End-point}

Patients were followed up in the outpatient clinic every 3 months. The end-point of the study was time to the first-event, either cardiovascular mortality or hospitalization for heart failure.

\section{Statistical analysis}

The normal distribution of the continuous variables was assessed with the Shapiro-Wilks test. In the event that a variable did not follow a normal distribution, a $\log _{2}$ transformation was performed, rechecking the normality with the same test. Continuous variables are shown as mean \pm standard deviation or median and interquartile range. Categorical variables are shown as absolute numbers and percentages with respect to the total. Comparison between continuous variables was done with the Student's t-test, and comparison of categorical variables was done with the chi-squared test without continuity correction. Linear regression analysis was done with the least squares method (r-Pearson). A Kaplan-Meier analysis was applied to plot the survival curves, and the comparison between them was done with the log-rank test. A multivariate Cox proportional risk model was used to evaluate the influence of blood levels of syndecan-1 in the outcomes of the patients. The covariates included were prespecified: age, diabetes, ischemic etiology, functional class, sinus rhythm, left ventricular ejection fraction (as continuous), NT-proBNP, and hsTn. In the event that colinearity was detected or the covariate did not modify the association between syndecan-1 and the outcome, a variable of the model could be excluded if no significant modification of -2log likelihood occurred. The R-statistical software was used to manage the data, and a p-value $<0.05$ was considered statistically significant.

\section{Results}

Ninety-five patients with heart failure and 24 matched healthy controls were included. Patients had higher levels of syndecan-1 (log transformed) than controls: 3.74 (SD 1.53) ng/mL vs. 2.84 (SD 1.7) ng/ $\mathrm{mL}, \mathrm{p}=0.01$.

The baseline characteristics of patients with heart failure are shown in Table 1. Thirty-eight patients (40\%) had experienced admission for heart failure in the year before inclusion in the study, with most of them (94.7\%) only having experienced one episode of hospitalization. Most of the included patients had a left ventricular ejection fraction below $50 \%(n=87,92 \%)$, and eight had a preserved ejection fraction. The mean ejection fraction of the whole group was $32 \%$ (SD 12). The majority of patients were in NYHA functional class II $(n=61,64 \%)$, 33 were in functional class III, and 1 was in functional class IV. As such, we allocated 61 patients (64.2\%) into a group labeled "good functional status" (class II) and 34 patients (35.8\%) into a group labeled "poor functional status" (class III and IV). We did not find significant differences in blood levels of $\log _{2}$ (syndecan-1) between patients with good and poor functional status: 3.68 (DE 1.72) ng/mL vs. 3.85 (DE 1.12) $\mathrm{ng} / \mathrm{mL}, \mathrm{p}=0.543$.

\section{Relationship with other biomarkers}

We did not find a significant linear relationship between $\log _{2}$ (syndecan-1) and hs-CRP $(\mathrm{p}=0.886), \mathrm{ST} 2(\mathrm{p}=0.776), \mathrm{hs} \operatorname{Tn}(\mathrm{p}=0.176)$ or NT-proBNP ( $\mathrm{p}=0.289)$. In the group of patients with measurable blood levels of IL1- $\beta$, a modest, although significant, linear relationship was found $(n=30 ; r=0.47, p=0.009)$. This relationship was also significant with the blood levels of IL-6 $(n=87 ; r=0.44, p<0.001)$ (Figure 1).

\section{Survival analysis}


The median follow-up was 544 days (interquartile range 285-688 days). During this period, 24 patients (25.2\%) died or were hospitalized due to heart failure. Patients were segregated into two groups, those with a value of $\log _{2}$ (syndecan-1) below and above the median $(3.78 \mathrm{ng} /$ $\mathrm{mL}$ ). We did not find significant differences in the time-event curves between both groups ( $\mathrm{p}=0.857$, Figure 2).

In the prespecified Cox regression model, functional status, sinus rhythm, and blood levels of NT-proBNP were finally removed due to colinearity or absence of confounding effect. The final model (Table 2) had a $-2 \log$ likelihood of $31.45(\mathrm{p}<0.001)$. $\log _{2}$ (syndecan-1) was significantly associated with the end-point with $\mathrm{HR}=0.64(0.45-0.92)$, $\mathrm{p}=0.015$

\section{Discussion}

The main findings of the study are: 1) blood concentrations of syndecan-1 are linearly correlated with some markers of systemic inflammation, such as IL-1 $\beta$ and IL-6, and 2) high levels of syndecan-1 are independently associated with a lower risk of death and hospitalization for heart failure in the mid-term follow-up. These results apply to stable heart failure patients with a broad range of left ventricular ejection fraction, mainly in functional classes II and III.

The information on syndecan-1 as a biomarker in patients with heart failure is scarce. Recently, Tromp et al. [7] reported the results from a study of 567 patients, $19 \%$ of them with preserved ejection fraction. They found an association between syndecan-1 and some remodeling biomarkers, but, contrary to our results, not with inflammatory markers. However, this does not invalidate our findings.

\begin{tabular}{|c|c|}
\hline Risk factors & Values \\
\hline Age (years) & $70 \pm 11$ \\
\hline Gender (males), n (\%) & $67(71)$ \\
\hline Arterial hypertension, $\mathrm{n}(\%)$ & $63(66)$ \\
\hline Current smoker, n (\%) & $9(9)$ \\
\hline Diabetes mellitus, n (\%) & $43(45)$ \\
\hline \multicolumn{2}{|l|}{ Previous history } \\
\hline Ischemic etiology, $\mathrm{n}(\%)$ & $37(40)$ \\
\hline Previous $\mathrm{PCl}, \mathrm{n}(\%)$ & $14(16)$ \\
\hline Previous CABG, n (\%) & $15(17)$ \\
\hline \multicolumn{2}{|l|}{ Heart rhythm } \\
\hline Sinus rhythm & $64(68)$ \\
\hline \multicolumn{2}{|l|}{ Echocardiographic data } \\
\hline End-diastolic volume (mL) & $175 \pm 73$ \\
\hline End-systolic volume (mL) & $125 \pm 68$ \\
\hline Ejection fraction (\%) & $32 \pm 12$ \\
\hline Ejection fraction $>50 \%, \mathrm{n}(\%)$ & $8(8)$ \\
\hline Absent or mild mitral regurgitation, $\mathrm{n}(\%)$ & $59(63)$ \\
\hline Moderate mitral regurgitation, n (\%) & $21(23)$ \\
\hline Severe mitral regurgitation, $\mathrm{n}(\%)$ & $13(14)$ \\
\hline \multicolumn{2}{|l|}{ Medications } \\
\hline$\beta$-blockers, n (\%) & $91(96)$ \\
\hline Angiotensin converting enzyme inhibitors, $\mathrm{n}(\%)$ & $66(69)$ \\
\hline Antagonists of angiotensin II receptors, n (\%) & $19(20)$ \\
\hline Spironolactone, $\mathrm{n}(\%)$ & $25(26)$ \\
\hline Eplerenone, n (\%) & $43(45)$ \\
\hline Diuretics, n (\%) & $93(98)$ \\
\hline Statins, n (\%) & $54(57)$ \\
\hline \multicolumn{2}{|l|}{ Devices } \\
\hline Cardiac resynchronization therapy, n (\%) & $10(11)$ \\
\hline Implantable cardioverter defibrillator, $\mathrm{n}(\%)$ & $21(22)$ \\
\hline
\end{tabular}

Table 1: Baseline characteristics of heart failure patients.
All of our patients were in a stable condition, and the fact that the patients recruited by Tromp et al. were included just before discharge after an acute heart failure admission could have influenced the results since, as the authors stated, a complete recovery at the time of sampling cannot be granted. Moreover, shedding of the glycocalyx is related to the activation of metalloproteinases [8] which in turn are activated by some inflammatory cytokines. As such, a positive relationship between syndecan-1 and inflammatory biomarkers is plausible.

Blood levels of syndecan-1 have been associated with the outcomes of patients with acute myocardial infarction [9], especially in patients with cardiogenic shock $[9,10]$ : Patients with higher levels of syndecan-1 had worse prognosis. In patients with heart failure, Neves et al. [11] reported a positive association between syndecan-1 and cardio-renal syndrome type 1 and the 6-month mortality rate, and Tromp et al. [7] reported a high risk of mortality and rehospitalization for heart failure, especially if the ejection fraction is preserved. The authors hypothesize that syndecan- 1 is a marker of endothelial damage and disruption and, as such, leads to a more compromised hemodynamic state, which, in turn, is related to prognosis. In our study, and contrary to the reported data, we found an inverse and highly significant relationship between syndecan-1 blood concentrations and outcomes (mortality and hospitalization for heart failure). Although blood concentrations of syndecan-1 can be related to the shedding of endothelial glycocalyx in acute situations, a protective effect in chronic states cannot be discarded. Lower levels of syndecan-1 are associated with less myocardial fibrosis and the ecto-domain of this proteoglycan retains binding properties reducing the bioavailability of syndecan-1 receptor ligands. As such, our findings should not be interpreted as a contradiction of the previous reports.

Endothelial glycocalyx has multiple biological functions. Among them, it reduces vascular permeability, prevents interaction of platelets and leukocytes, acts as a sodium buffer and is also a mechano-transducer [12-15]. Oxidative stress, ischemia and reperfusion, inflammation, and even natriuretic peptides can potentially damage and shed the glycocalyx [16-18], leading to a diffuse endothelial dysfunction and tissue edema. Sodium can bind to glycosaminoglycans, a main component of the interstitium linked to the proteoglycans [19-21], creating a hypertonic microenvironment and promoting lymphangiogenesis. If this interstitial glycosaminoglycan network becomes dysfunctional, the compliance of the interstitium may be diminished and tissue edema can ensue [5]. Edema is related to organ dysfunction (even in the myocardium) and tissue fibrosis $[22,23]$. This process can contribute to heart failure decompensation and to the progression of chronic heart failure.

Some other glycocalyx shedding makers have been proposed. Syndecan-4 has been studied in patients with heart failure [24,25]. Although a moderate value to discriminate patients who are rehospitalized for heart failure has been reported, the results not have been replicated by other investigators.

This study had some limitations. First, the sample size was small

\begin{tabular}{|c|c|c|c|}
\hline Factor & Hazard ratio & CI 95\% & p \\
\hline Log $_{2}$-syndecan-1 & 0.64 & $0.45-0.92$ & 0.015 \\
\hline Age & 1.04 & $0.99-1.10$ & 0.134 \\
\hline Diabetes & 8.04 & $2.57-25.17$ & $<0.001$ \\
\hline Ischemic etiology & 0.58 & $0.22-1.52$ & 0.271 \\
\hline Ejection fraction & 0.95 & $0.91-0.99$ & 0.042 \\
\hline Hs-Troponin T & 1.04 & $1.00-1.08$ & 0.037 \\
\hline
\end{tabular}

Note: $\mathrm{Cl}$ : Confidence Interval. Non-significant variables are retained as modifiers.

Table 2: Cox proportional regression model. 


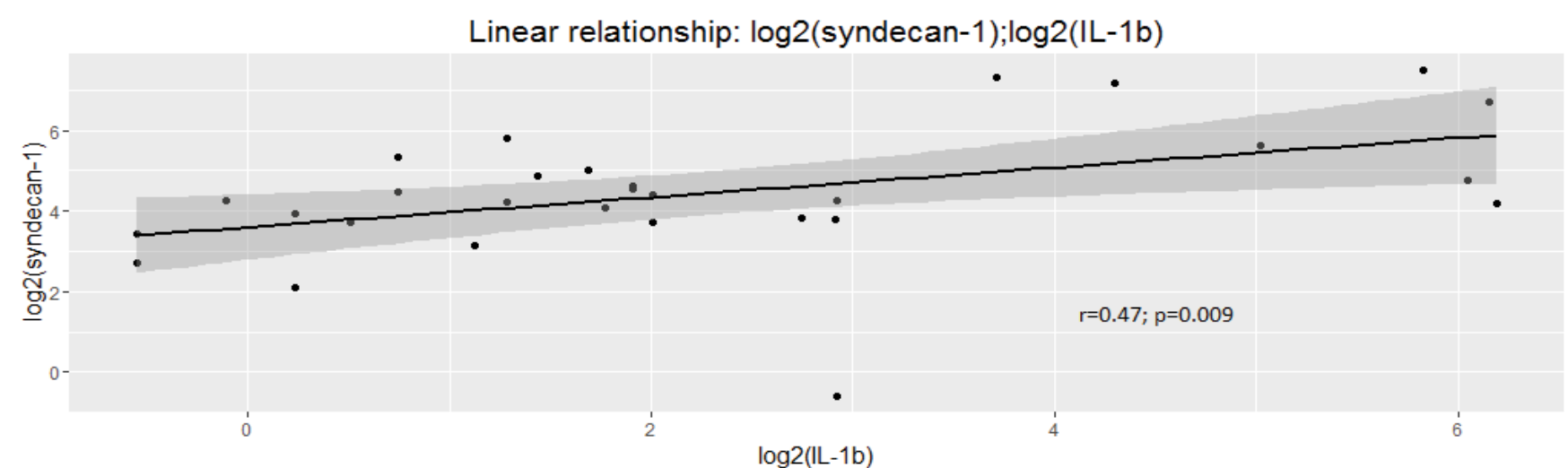

Linear relationship: $\log 2($ syndecan-1);log2(IL-6)

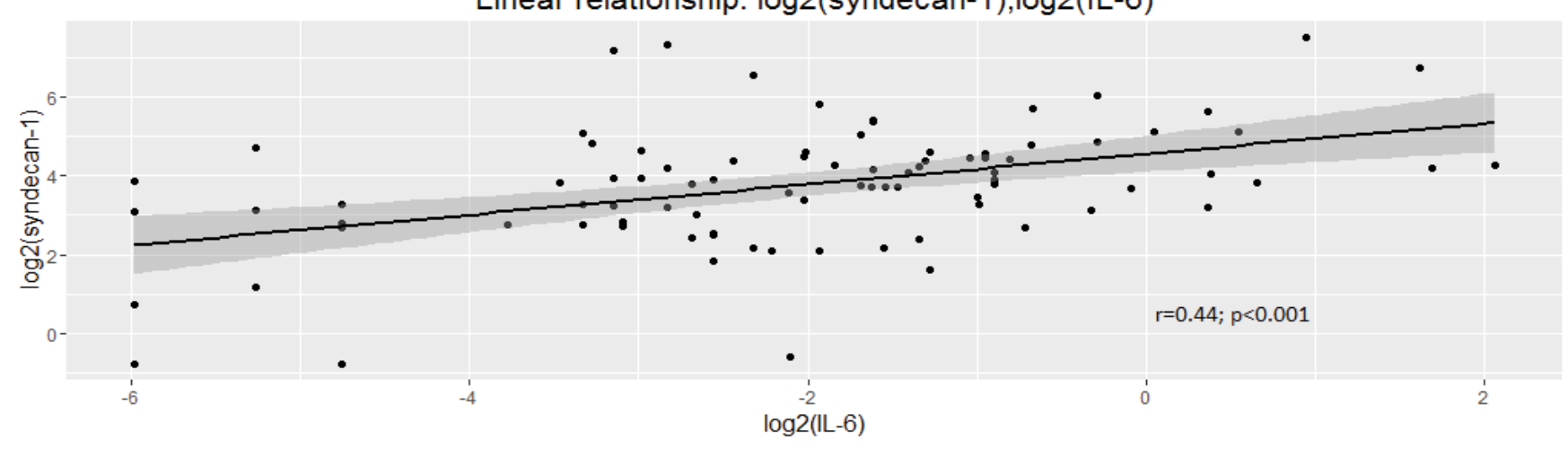

Figure 1: Log-log linear relationships between $\log _{2}$ (syndecan-1) and $\log _{2}-\mathrm{IL}-1 \beta$ and $\log _{2}-\mathrm{IL}-6$.

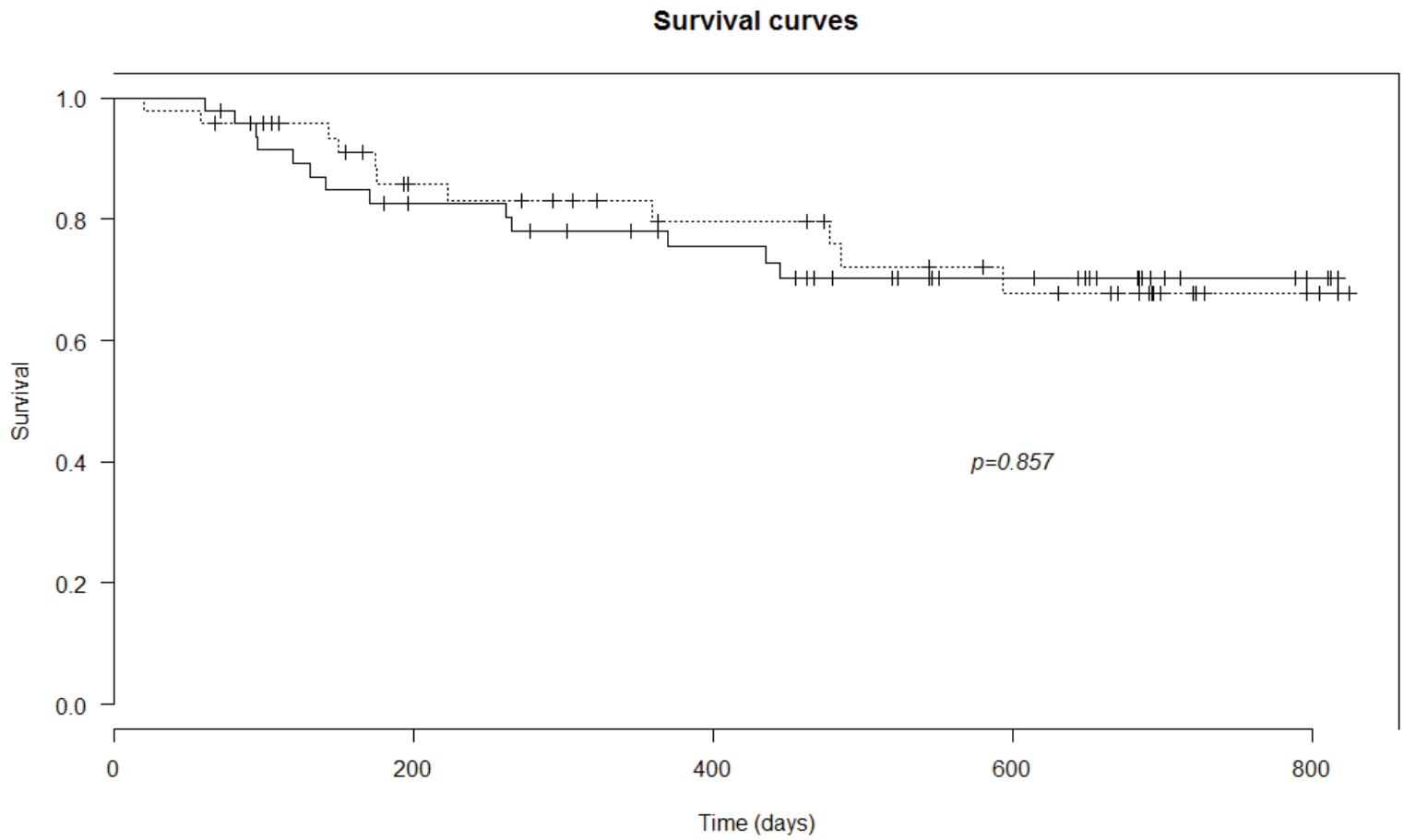

Figure 2: Survival curves. Dotted line: $\log _{2}($ syndecan-1) above the median. 
Citation: Egido AGD, Iglesias-Garriz I, Rodriguez-Garcia MA, Fernandez-Vazquez F, Gonzalo-Orden JM (2018) Syndecan-1 and Outcome of Ambulatory Patients with Stable Heart Failure. J Mol Biomark Diagn 9: 401. doi: 10.4172/2155-9929.1000401

and therefore a subgroup analysis was not realized. Second, patient enrollment was performed from a heart failure unit where most of the patients evaluated were potential candidates for advanced therapies, such as cardiac resynchronization, transplantation or ventricular assists, and that is the reason for patients with preserved ejection fraction were underrepresented in our study. And third, the results of this investigation are referenced to patients with a rigorous adjustment of the treatment, based on current scientific evidence, and may not be representative for the entire population with heart failure.

\section{Conclusion}

In conclusion, in patients with stable heart failure, blood concentrations of syndecan-1 are correlated to pro-inflammatory cytokines. Higher levels of syndecan-1 are independently associated with a lower probability of cardiovascular death and hospitalization for heart failure in the mid-term follow-up. However, we acknowledge that our results should be confirmed in larger studies, since previous reports suggest that high syndecan-1 blood levels are associated with a worse outcome in patients with acute heart failure or preserved ejection fraction.

\section{Acknowledgments}

The authors acknowledge the invaluable assistance received from Ms. Trinidad Verduras de la Varga, nurse of heart failure unit, thanks to which it has been possible to carry out the present study.

\section{Author Declaration}

All the authors take responsibility for all aspects of the reliability and freedom from bias of the data presented and their discussed interpretation. This work has not received grants from any public or private entity. There are no conflicts of interest.

\section{References}

1. Remes J, Miettinen H, Reunanen A, Pyorala K (1991) Validity of clinical diagnosis of heart failure in primary health care. Eur Heart J 12: 315-321.

2. Wheeldon NM, MacDonald TM, Flucker CJ, McKendrick AD, McDevitt DG, et al. (1993) Echocardiography in chronic heart failure in the community. Q J Med 86: $17-23$

3. Kantor PF, Rusconi P, Lipshultz S, Mital S, Wilkinson JD, et al. (2011) Current applications and future needs for biomarkers in pediatric cardiomyopathy and heart failure: Summary from the second international conference on pediatric cardiomyopathy. Prog Pediatr Cardiol 32: 11-14.

4. Braunwald E (2008) Biomarkers in heart failure. N Engl J Med 358: 2148-2159.

5. Nijst P, Verbrugge FH, Grieten L, Dupont M, Steels P, et al. (2015) The pathophysiological role of interstitial sodium in heart failure. J Am Coll Cardiol 65: $378-388$

6. Alexopoulou AN, Multhaupt HAB, Couchman JR (2007) Syndecans in wound healing, inflammation and vascular biology. Int J Biochem Cell Biol 39: 505-528.

7. Tromp J, Van Der Pol A, Klip IT, De Boer RA, Jaarsma T, et al. (2014) Fibrosis marker syndecan- 1 and outcome in patients with heart failure with reduced and preserved ejection fraction. Circ Heart Fail 7: 457-462.

8. Endo K, Takino T, Miyamori H, Kinsen H, Yoshizaki T, et al. (2003) Cleavage of syndecan-1 by membrane type matrix metalloproteinase-1 stimulates cell migration. J Biol Chem 278: 40764-40770.

9. Ostrowski SR, Pedersen SH, Jensen JS, Mogelvang R, Johansson PI (2013) Acute myocardial infarction is associated with endothelial glycocalyx and cell damage and a parallel increase in circulating catecholamines. Crit Care 17: R32.

10. Jung C, Fuernau G, Muench P, Desch S, Eitel I, et al. (2015) Impairment of the endothelial glycocalyx in cardiogenic shock and its prognostic relevance. Shock 43: 450-455.

11. Neves FM, Meneses GC, Sousa NE, Menezes RR, Parahyba MC, et al. (2015) Syndecan-1 in acute decompensated heart failure-association with renal function and mortality. Circ J 79: 1511-1519.

12. Salmon AH, Satchell SC (2012) Endothelial glycocalyx dysfunction in disease: Albuminuria and increased microvascular permeability. J Pathol 226: 562-574.

13. Reitsma S1, Oude Egbrink MG, Heijnen VV, Megens RT, Engels W, et al. (2011) Endothelial glycocalyx thickness and platelet-vessel wall interactions during atherogenesis. Thromb Haemost 106: 939-946

14. Korte S, Wiesinger A, Straeter AS, Peters W, Oberleithner H, et al. (2012) Firewall function of the endothelial glycocalyx in the regulation of sodium homeostasis. Pflugers Arch 463: 269-278.

15. Florian JA, Kosky JR, Ainslie K, Pang Z, Dull RO, et al. (2003) Heparan sulfate proteoglycan is a mechano-sensor on endothelial cells. Circ Res 93: e136-42.

16. Reitsma S, Slaaf DW, Vink H, Van Zandvoort MAMJ, Oude Egbrink MGA (2007) The endothelial glycocalyx: Composition, functions, and visualization. Pflugers Arch 454: 345-359.

17. Kurzelewski M, Czarnowska E, Beresewicz A (2005) Superoxide- and nitric oxide-derived species mediate endothelial dysfunction, endothelial glycocalyx disruption, and enhanced neutrophil adhesion in the post-ischemic guinea-pig heart. J Physiol Pharmacol 56: 163-178.

18. Jacob M, Saller T, Chappell D, Rehm M, Welsch U, et al. (2013) Physiological levels of A-, B- and C-type natriuretic peptide shed the endothelial glycocalyx and enhance vascular permeability. Basic Res Cardiol 108: 347

19. Titze J, Machnik A (2010) Sodium sensing in the interstitium and relationship to hypertension. Curr Opin Nephrol Hypertens 19: 385-392.

20. Wiig H, Swartz MA (2012) Interstitial fluid and lymph formation and transport Physiological regulation and roles in inflammation and cancer. Physiol Rev 92 1005-1060.

21. Szabo G, Magyar Z (1982) Electrolyte concentrations in subcutaneous tissue fluid and lymph. Lymphology 15: 174-177.

22. Dongaonkar RM, Stewart RH, Geissler HJ, Laine GA (2010) Myocardial microvascular permeability, interstitial oedema, and compromised cardiac function. Cardiovasc Res 87: 331-339.

23. Garcia-Dorado D, Andres-Villarreal M, Ruiz-Meana M, Inserte J, Barba I (2012) Myocardial edema: A translational view. J Mol Cell Cardiol 52: 931-939.

24. Takahashi R, Negishi K, Watanabe A, Arai M, Naganuma F, et al. (2011) Serum syndecan- 4 is a novel biomarker for patients with chronic heart failure. J Cardiol 57: 325-332.

25. Bielecka-Dabrowa A, Von Haehling S, Aronow WS, Ahmed MI, Rysz J, et al. (2013) Heart failure biomarkers in patients with dilated cardiomyopathy. Int J Cardiol 168: 2404-2410. 